\title{
JMJD2C promotes colorectal cancer metastasis via regulating histone methylation of MALAT1 promoter and enhancing $\beta$-catenin signaling pathway
}

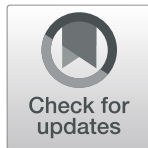

Xinnan $\mathrm{Wu}^{1 \dagger}$, Ruixiao $\mathrm{Li}^{1 \dagger}$, Qing Song ${ }^{1,2 \dagger}$, Chengcheng Zhang ${ }^{3}, \mathrm{Ru} \mathrm{Jia}^{1}$, Zhifen Han ${ }^{1}$, Lihong Zhou ${ }^{1}$, Hua Sui ${ }^{1}$, Xuan Liu', Huirong Zhu' ${ }^{1}$, Liu Yang ${ }^{1}$, Yan Wang ${ }^{1}$, Qing $\mathrm{Ji}^{1 *}$ and Qi $\mathrm{Li}^{1,4^{*}}$

\begin{abstract}
Background: Our previous work demonstrated that IncRNA-MALAT1 was overexpressed in recurrent colorectal cancer (CRC) and metastatic sites in post-surgical patients. However, the upstream regulatory mechanism of MALAT1 is not well-defined. Histone demethylase JMJD2C holds great potential of epigenetic regulating mechanism in tumor diseases, especially the moderating effect on the promoter activity of targeted genes associated closely with tumor development. Therefore, we herein investigated whether JMJD2C could epigeneticly regulate the promoter activity of MALAT1 and the downstream $\beta$-catenin signaling pathway, thereby affecting the metastatic abilities of CRC cells.

Methods: JMJD2C expressions in human CRC samples were detected by real-time PCR and immunohistochemistry staining. Gene silencing and overexpressing efficiencies of JMJD2C were confirmed by real-time PCR and western blot. The migration of CRC cells in vitro were tested by transwell and wound healing assays. The protein expression and cellular localization of JMJD2C and $\beta$-catenin were characterized by immunofluorescence staining and western blot. The histone methylation level of MALAT1 promoter region (H3K9me3 and H3K36me3) was tested by ChIP-PCR assays. The promoter activity of MALAT1 was detected by luciferase reporter assay. The expressions of MALAT1 and the downstream $\beta$-catenin signaling pathway related genes in CRC cells were detected by real-time PCR and western blot, respectively. The nude mice tail vein metastasis model was established to observe the effect of JMJD2C on the lung metastasis of CRC cells in vivo.

Results: Our present results indicated that histone demethylase JMJD2C was overexpressed in matched CRC tumor tissues of primary and metastatic foci, and CRC patients with lower JMJD2C expression in primary tumors had better prognosis with longer OS (Overall Survival). The following biological function observation suggested that JMJD2C promoted CRC metastasis in vitro and in vivo. Further molecular mechanism investigation demonstrated that JMJD2C protein translocated into the nuclear, lowered the histone methylation level of MALAT1 promoter in the sites of H3K9me3 and H3K36me3, up-regulated the expression of MALAT1, and enhanced the $\beta$-catenin signaling pathway in CRC cells.

(Continued on next page)
\end{abstract}

\footnotetext{
* Correspondence: ttt99118@hotmail.com; qili@shutcm.edu.cn

${ }^{+}$Xinnan Wu, Ruixiao Li and Qing Song contributed equally to this work.

1 Department of Medical Oncology and Cancer Institute of Medicine,

Shuguang Hospital, Shanghai University of Traditional Chinese Medicine,

Shanghai, China

Full list of author information is available at the end of the article
}

(c) The Author(s). 2019 Open Access This article is distributed under the terms of the Creative Commons Attribution 4.0 International License (http://creativecommons.org/licenses/by/4.0/), which permits unrestricted use, distribution, and reproduction in any medium, provided you give appropriate credit to the original author(s) and the source, provide a link to the Creative Commons license, and indicate if changes were made. The Creative Commons Public Domain Dedication waiver (http://creativecommons.org/publicdomain/zero/1.0/) applies to the data made available in this article, unless otherwise stated. 
(Continued from previous page)

Conclusion: Our data demonstrated that JMJD2C could enhance the metastatic abilities of CRC cells in vitro and in vivo by regulating the histone methylation level of MALAT1 promoter, thereby up-regulating the expression of MALAT1 and enhancing the activity of $\beta$-catenin signaling pathway, providing that JMJD2C might be a novel therapeutic target for CRC metastasis.

Keywords: Histone demethylase JMJD2C, MALAT1, $\beta$-Catenin signaling pathway, Colorectal cancer, Metastasis

\section{Background}

Colorectal cancer (CRC) is one of the most common causes of cancer death, bringing much inconvenience to people's daily life [1]. Although an increasing number of new therapy methods are applied to CRC treatment, the therapeutic efficacy for advanced CRC patients is not good. Tumor recurrence or metastasis is still the main cause of therapy failure [2] and poor prognosis. The 5year survival rates of CRC patients were about $90 \%$ for the ones with only primary tumors, $70.4 \%$ for the ones with lymph node or peripheral metastasis, and $12.5 \%$ for the ones with distal metastasis [3].

Long non-coding RNAs (lncRNAs) have been discovered to act important roles in tumor development [4]. Metastasis associated in lung adenocarcinoma transcript 1 (MALAT1) is one of these functional lncRNAs, which is highly expressed in CRC patients. In our previous studies, we have successfully demonstrated that MALAT1 could promote tumor growth and metastasis in CRC by binding to SFPQ (PTB-associated splicing factor) and releasing oncogene PTBP-2 (polypyrimidine tract binding protein) from the SFPQ/PTBP-2 complex [5], and increase the nuclear translocation of $\beta$-catenin from cytoplasm, thereby activating downstream genes of $\beta$-catenin signaling pathway $[6,7]$. Ying et al also revealed that, in bladder cancer, MALAT1 promoted EMT by activating Wnt signaling in vitro [8]. However, the upstream regulatory mechanism of MALAT1 is not well-elucidated.

Jumonji domain-containing protein 2C (JMJD2C), also known as KDM4C, could modulate transcription factors, establish global chromatin environments and regulate gene expression [9-11]. A number of evidences have suggested the association between JMJD2C protein and various tumors [12-15]. Histone demethylase JMJD2C holds great potential of epigenetic regulating mechanism in tumor diseases $[16,17]$, especially the regulating effect on the promoter activity of targeted genes. Based on bioinformatics analysis of the MALAT1 promoter and our previous studies, we hypothesized that, JMJD2C might influence MALAT1 promoter activity, thereby activating MALAT1/ $\beta$-catenin signaling pathway and leading to the promotion of CRC metastasis.

Therefore, in this study, we aimed to investigate the new effect of JMJD2C on the metastasis of CRC cells in vitro and in vivo, and to detect the regulatory effect of
JMJD2C on the expression of MALAT1 and $\beta$-catenin signaling pathway related genes. Most importantly, the potential epigenetic regulating mechanism of JMJD2C on MALAT1 was the focus of this research.

\section{Materials and methods}

Human tissues collection and cell culture

A total of 78 human primary CRC tissues without metastasis and 46 human primary CRC tissues with matched hepatic or lung metastasis were collected between 2006 and 2015 at Shuguang Hospital, Shanghai University of Traditional Chinese Medicine, and Fudan University Shanghai Cancer Center. HCT116 (human colon, CRC) and LoVo (human colon, Dukes' type C, grade IV, colorectal adenocarcinoma) cells were purchased from the ATCC (Manassas, USA), and cultured in RPMI-1640 and F12K medium, respectively. Both mediums were supplemented with $10 \%$ heat-inactivated FBS, $100 \mathrm{U} \mathrm{ml}^{-1}$ penicillin, and $100 \mu \mathrm{g} \mathrm{ml}^{-1}$ streptomycin. These cells were incubated under $37^{\circ} \mathrm{C}, 5 \% \mathrm{CO}_{2}$ conditions. All the experimental procedures were approved by the Institutional Review Board of Shuguang Hospital, Shanghai University of Traditional Chinese Medicine.

\section{Plasmids construction and lentivirus infection}

Three shRNA fragments for human JMJD2C (Gene ID: 23081) were synthesized by the Sangon Biotech company (Shanghai, China), sub-cloned into the pLKD shRNA vector, and named pLKD-CMV-G\&PR-U6JMJD2C-shRNA1/2/3, respectively. The full length of JMJD2C gene was amplified by PCR, following by gene sequencing, and the right full fragments was cloned into the plenti overexpression vector, named plenti-CMVEGFP-P2A-JMJD2C-3FLAG. These above plasmids were used to transfect well-growing state HCT116 and LoVo cells by using Lipofectamine 3000 transfection reagent. After $48 \mathrm{~h}$ transfection, the transfected cells were selected with neomycin (G418, $1 \mathrm{mg} \mathrm{ml}^{-1}$, Sigma, USA). Recombinant lentiviruses containing pLV4-shRNA/NT, pLV4-shRNA/JMJD2C, pLV4-empty vector, and pLV4JMJD2C ${ }^{+/+}$were prepared by GeneChem (Shanghai, China), respectively. HCT116 or LoVo cells were infected with $2 \times 10^{6}$ transducing units of corresponding lentiviruses, and were selected with $2 \mu \mathrm{g} / \mathrm{ml}$ puromycin 
for 2 weeks. The efficiencies of knockdown or overexpression of JMJD2C were determined by real-time PCR and western blot.

\section{Real-time PCR}

Total RNA was extracted using the TRIzol reagent (Takara) according to the manufacturer's instructions. The RNA concentrations were determined using a NanoDrop ND-1000 (NanoDrop). cDNA was synthesized with the PrimeScript RT Reagent Kit (TaKaRa) using $500 \mathrm{ng}$ total RNA as template. Real-time PCR analyses were conducted to quantitate KDM4C mRNA and IncRNAMALAT1 relative expression using SYBR Premix Ex Taq (TaKaRa) with GAPDH as an internal control. The Realtime PCR results were defined from the threshold cycle $(\mathrm{Ct})$, and relative expression levels were calculated by using the $2^{-\triangle \Delta C t}$ method. PCR was performed using an ABI 7500 instrument (Applied Biosystems, USA). The primers used for real-time PCR analysis were as follow: KDM4C, forward primer: 5-AGGCCTAAGGCTGA TGAGGA-3, reverse primer: 5-TTGGCCATGAAAGC TCGGAT-3; MALAT1, forward primer: 5-GCTCTG TGGTGTGGGATTGA-3, reverse primer: 5-GTGGCA AAATGGCGGACTTT-3, GAPDH, forward primer: 5GGTGGTCTCCTCTGACTTCAACA-3, reverse primer: 5-CCAAATTCGTTGTCATACCAGGAAATG-3.

\section{Luciferase reporter assay}

To test MALAT1 promoter activity, HCT116 or LoVo cells were co-transfected with the recombinant plasmid pGL3-basic-MALAT1 promoter with a control positive plasmid pRL-SV40 as previously described [18]. The promoter activity was analyzed using a commercial dualluciferase assay kit (Promega, USA) according to the manufacturer's instructions.

\section{Immunofluorescence staining}

To observe the expression and location of JMJD2C and $\beta$-catenin, HCT116 and LoVo cells after transfection were plated at a density of $2.0 \times 10^{4} / \mathrm{ml}$ in 6 -well plates, fixed with methanol, blocked with $5 \%$ BSA. The cells were first stained with $\beta$-catenin mouse antibody followed by Cy3-conjugated goat anti-mouse IgG (Millipore). After the cells were washed four times with PBS, the JMJD2C rabbit antibody was added, followed by FITC-conjugated goat anti-rabbit IgG (Millipore). Nuclear staining was done with 4',6-diamidino-2-phenylindole dihydrochloride (DAPI) solution. Cells were imaged using TCS SP2 spectral confocal system (Leica, Germany). All experiments were conducted according to instructions from the antibody manufacturer.

\section{Western blot analysis}

Protein lysates from cells were prepared in lysis buffer and centrifuged at $12000 \mathrm{rpm}$ at $4{ }^{\circ} \mathrm{C}$. The primary antibodies used were JMJD2C (Santa Cruz, USA), $\beta$-catenin (CST, USA), c-Myc (CST, USA), ITGBL1 (Abcam, USA), PCNA (CST, USA) and GAPDH (CST, USA). The secondary antibody used was HRP-labeled goat anti-rabbit/mouse IgG $(\mathrm{H}+\mathrm{L})$ (Beyotime, China). Each band was quantitatively analyzed using quantity one Software and normalized to the expression of GAPDH in the same lane.

\section{Chromatin immunoprecipitation assay}

ChIP assays were performed using a ChIP assay kit (Millipore, USA). Chromatin extracts were immunoprecipitated using $10 \mu \mathrm{g}$ anti-JMJD2C (Abcam, USA), $4 \mu \mathrm{g}$ anti-H3K9 (Abcam, USA), and $4 \mu \mathrm{g}$ anti-H3K36 (Abcam, USA), respectively. IgG (Merck, Germany) were used as mock ChIP controls. Fold enrichments were calculated by determining the ratios of the amount of immunoprecipitated DNA to that of the input sample, and were normalized to the level observed at a control region, which was defined as 1.0. The ChIP primers for MALAT1 promoter are as follow: forward primer: 5GGTCAGCCTGAGACCACTTC-3, reverse primer: 5CTGTGCCTGTTCTGGGGAAT-3.

\section{Transwell assay}

To measure cell migration, the transfected HCT116 and LoVo cells $\left(2 \times 10^{5}\right)$ were seeded to the upper chambers and cultured with $100 \mu \mathrm{l}$ serum-free F12K or RPMI1640 medium, whereas the lower chamber was filled with $600 \mu \mathrm{l} \mathrm{F12K}$ or RPMI1640 medium containing 15\% FBS and $10 \mu \mathrm{g} \mathrm{ml}^{-1}$ fibronectin. After incubation for $48 \mathrm{~h}$ at $37{ }^{\circ} \mathrm{C}$ and $5 \% \mathrm{CO}_{2}$, the chambers were fixed with $4 \%$ paraformaldehyde and stained with $0.1 \%$ crystal violet. The cells in the upper chamber were carefully removed with a cotton swab. The numbers of cells were analyzed by counting five independent visual fields under a DMI3000 B inverted microscope (Leica, USA) with a $20 \times$ objective. All assays were performed in triplicate and independently repeated three times.

\section{Wound healing assay}

Cells $\left(4.5 \times 10^{5}\right)$ were seeded on a six-well plate to form a confluent monolayer in 10\% FBS-containing medium. The monolayer cells were scratched by a plastic tip and washed with PBS to remove cell debris; 0.5\% FBS-containing F12K or RPMI1640 were then added to each well, and the scratched monolayer was incubated in a $37^{\circ} \mathrm{C}$ incubator with $5 \% \mathrm{CO}_{2}$ for $24 \mathrm{~h}$. Wound closure was measured in five random fields at $\times 200$ magnification using Image J software and a DMI3000B inverted microscope (Leica, USA). Percentage of wound healing was calculated as 
follows: migrated cell surface area/ total surface area $x$ 100 , in which, migrated cell surface area $=$ length of cell migration $(\mathrm{mm}) \times 2 \times$ length of defined areas, total surface area $=$ beginning width $\times$ length of defined areas.

\section{MTT assay}

Cells were cultured at a density of $2.5 \times 10^{3}$ cells per well in a flat-bottomed 96-well plate. MTT [3-(4,5)dimethylthiahiazo(-z-y1)-3,5-di-phenytetrazoliumromide] was used to determine the cell viability by measuring the absorbance at $490 \mathrm{~nm}$. All assays were performed in triplicate and independently repeated three times.

\section{Flow cytometry}

Cells were harvested by trypsinization, washed once with cold PBS. Then, the cells were stained with PI (propidium iodide) and anti-Annexin- $\mathrm{V}$ antibody (Becton Dickinson, USA) at $4{ }^{\circ} \mathrm{C}$ for $1 \mathrm{~h}$. Subsequently, cells were washed once with PBS and analyzed by FACS (BD, USA).

\section{In vivo analysis}

All the animal experiments were performed under the approval of the Institutional Committee for Animal Research and were in accordance with national guidelines for the care and use of laboratory animals. HCT116shRNA/NT, HCT116-shRNA/JMJD2C, HCT116-empty vector, and HCT116-JMJD2C $\mathrm{C}^{+/+}$cells $\left(2 \times 10^{6}\right.$ cells in $100 \mu \mathrm{l})$ were injected into female BALB/c nude mice (68 weeks old, obtained from SLAC Laboratory Lab, Shanghai, China) by tail intravenous to establish the lung metastasis mice model, respectively. Mice were euthanized 42 days post injection, and the lung metastases

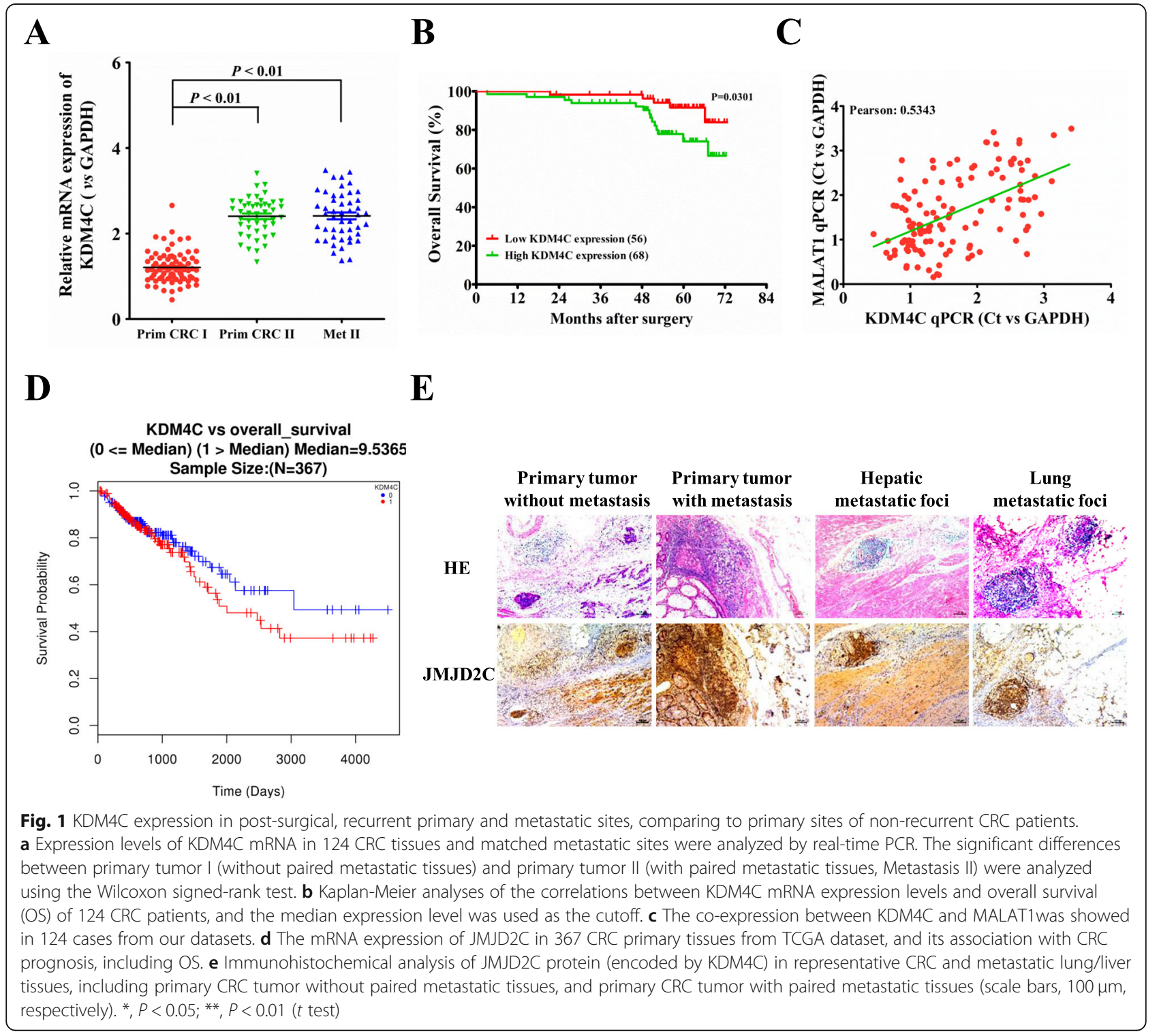


images were observed and quantified by LB983 NIGHTOWL II system. The numbers of lung metastatic nodes were measured, and the metastatic tumor tissues were collected for immunohistological and western blot analysis with JMJD2C, $\beta$-catenin, c-Myc, and ITGBL1 antibody.

\section{HE staining and IHC staining}

Paraffin-embedded tissues were sectioned for $\mathrm{HE}$ (hematoxylin and eosin) and IHC (immunohistochemistry) staining. For IHC analysis, the experiments were performed using the first antibody, HRP-conjugated secondary antibody, and DAB (diaminobenzidine) detection reagents. The DMI3000B microscope connected to the digital imaging system was applied for taking photographs and the following analysis. All the data were evaluated and classified blindly by two investigators (ZYW and LLW) from the pathology department of Shuguang Hospital, Shanghai University of Traditional Chinese Medicine.

\section{Statistical analysis}

All data are presented as means with standard deviation (SD) or median with $95 \%$ confidence interval (95\% CI). Statistic comparison was performed using the Student's t-test, one-way ANOVA analysis, Mann-Whitney test, or Kruskal-Wallis test, as appropriate, with the significance level at $P<0.05$. All statistical analyses were completed with SPSS 22 software.

\section{Results}

Overexpression of JMJD2C in CRC metastatic foci predicted poor prognosis

Firstly, the expression levels of KDM4C mRNA (encoding JMJD2C protein) were detected in 124 CRC tumor

Table 1 Association between KDM4C expression and clinicopathological variables of CRC patients

\begin{tabular}{|c|c|c|c|}
\hline \multirow[t]{2}{*}{ Variables } & Low KDM4C expression $(n=67)$ & High KDM4C expression $(n=57)$ & $P$ \\
\hline & n (\%) & n (\%) & \\
\hline \multicolumn{4}{|l|}{ Age } \\
\hline$>65$ & $13(19.40)$ & $10(17.54)$ & \multirow[t]{2}{*}{0.8210} \\
\hline$\leq 65$ & $54(80.60)$ & $47(82.46)$ & \\
\hline \multicolumn{4}{|l|}{ Gender } \\
\hline Male & $39(58.21)$ & $39(68.42)$ & \multirow[t]{2}{*}{0.2673} \\
\hline Female & $28(41.79)$ & $18(31.58)$ & \\
\hline \multicolumn{4}{|l|}{ Tumor site } \\
\hline Rectum & $37(55.22)$ & $36(63.16)$ & \multirow[t]{2}{*}{0.4642} \\
\hline Colon & $30(44.78)$ & $21(36.84)$ & \\
\hline \multicolumn{4}{|l|}{ Tumor differentiation } \\
\hline Well & $20(29.85)$ & 19 (33.33) & \multirow[t]{2}{*}{0.7019} \\
\hline Moderate + Poor & $47(70.15)$ & $38(66.67)$ & \\
\hline \multicolumn{4}{|l|}{ TNM stage } \\
\hline Stage II & $35(52.23)$ & $12(21.05)$ & \multirow[t]{2}{*}{$0.0004^{* *}$} \\
\hline Stage III & $32(47.77)$ & $45(78.95)$ & \\
\hline \multicolumn{4}{|c|}{ Lymph vascular invasion } \\
\hline Positive & $44(65.67)$ & $42(73.68)$ & \multirow[t]{2}{*}{0.4347} \\
\hline Negative & $23(34.33)$ & $15(26.32)$ & \\
\hline \multicolumn{4}{|l|}{ Perineural invasion } \\
\hline Positive & $52(77.61)$ & $46(80.70)$ & \multirow[t]{2}{*}{0.8253} \\
\hline Negative & $15(22.39)$ & $11(19.30)$ & \\
\hline \multicolumn{4}{|l|}{ Liver metastasis } \\
\hline Positive & $9(13.43)$ & $22(38.60)$ & \multirow[t]{2}{*}{$0.0017^{* *}$} \\
\hline Negative & $58(86.57)$ & $35(51.40)$ & \\
\hline \multicolumn{4}{|l|}{ Lung metastasis } \\
\hline Positive & $1(1.49)$ & $8(14.04)$ & \multirow[t]{2}{*}{$0.0114^{*}$} \\
\hline Negative & 66 (98.51) & 49 (85.96) & \\
\hline
\end{tabular}

${ }^{*}, P<0.05 ;{ }^{* *}, P<0.01$ 
specimens. The results demonstrated that, the levels of KDM4C mRNA were significantly higher in recurrent tumors and metastatic sites than that of non-recurrent ones (Fig. 1a), and the mRNA levels were closely associated with TMN stages and distant metastasis (Table 1). Survival analysis concluded that CRC patients with lower KDM4C mRNA expression had longer OS (Overall Survival) (Fig. 1b). In our previous reports, we have shown in $124 \mathrm{CRC}$ tumor specimens that MALAT1 had higher expression levels in recurrent primary and metastatic sites, relative to non-recurrent primary tumors (7). In the present study, we further found that, highly matched co-expression of KDM4C mRNA and MALAT1 was found in our 124 CRC cases (Fig. 1c). In addition, a TCGA (The Cancer Genome Atlas)-dataset analysis also showed the elevated mRNA levels of KDM4C in 367 CRC primary tissues, and the KDM4C mRNA expression was inversely correlated with OS of CRC patients
(Fig. 1d). Next, the immunohistochemistry staining further revealed significantly higher protein levels of JMJD2C (encoded by KDM4C) in recurrent tumors and lung/liver metastasis than the ones in non-recurrent tumors (Fig. 1e), implying that JMJD2C overexpression correlated closely with CRC progression.

\section{JMJD2C promoted the migration of CRC cell lines in vitro} To thoroughly understand the biological function of JMJD2C, we first knocked down and overexpressed JMJD2C expression using shRNA and overexpressing vector, respectively. Real-time PCR assay was carried out to detect the mRNA expression of JMJD2C and showed efficient downregulation or up-regulation of JMJD2C in HCT116 and LoVo cells (Fig. 2a, Additional file 1: Figure S1A). The protein expression of JMJD2C was further confirmed by western blot and quantitative assay (Fig. 2b, c, Additional file 1: Figure S1B, C). Subsequently, by transwell and wound

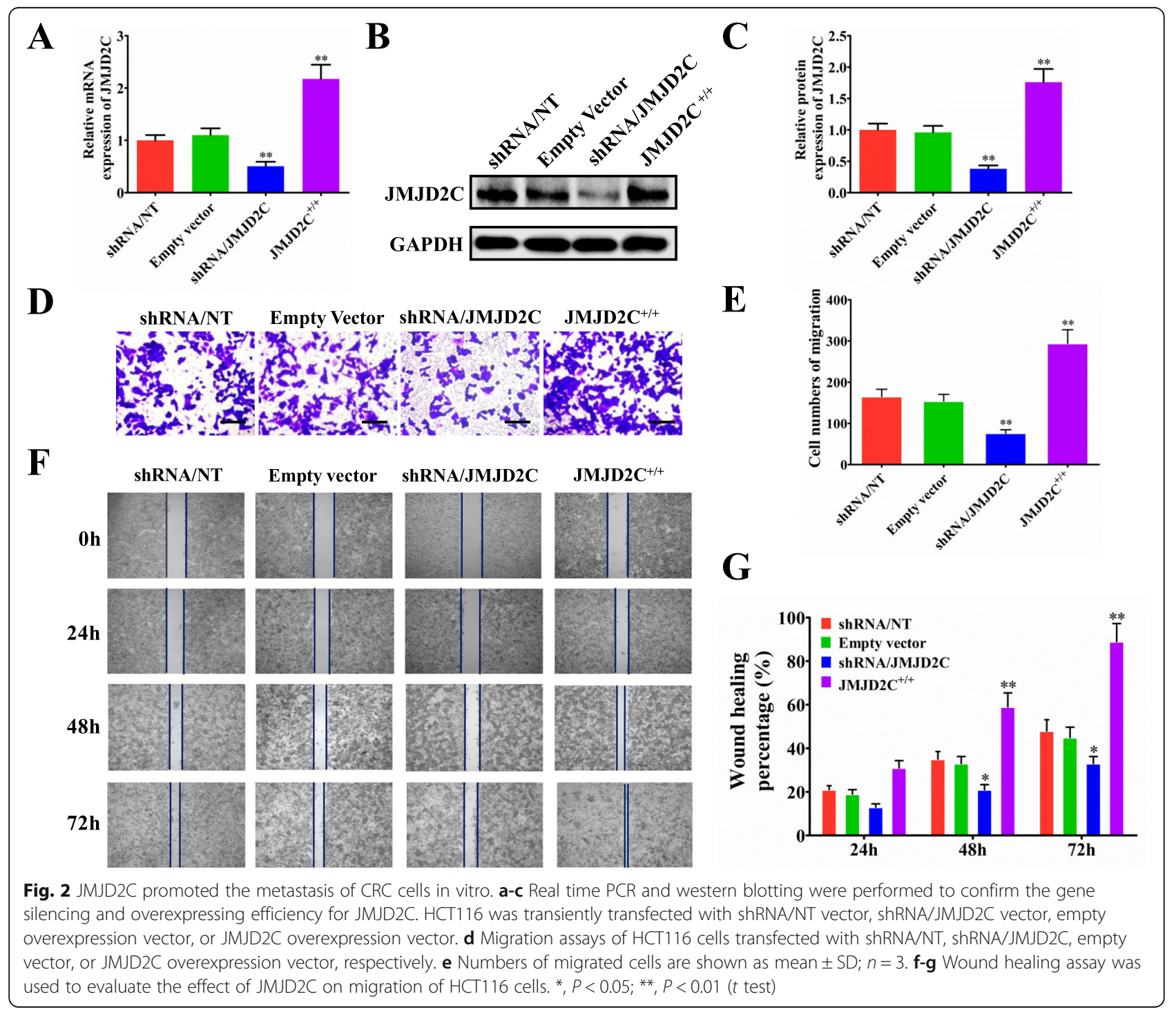


healing assays in HCT116 and LoVo cells, we found a positive role of JMJD2C in promoting CRC cells migration (Fig. 2d-g, Additional file 1: Figure S1D, E). Additionally, we also found that, JMJD2C could promote the proliferation of CRC cells (Additional file 2: Figure S2A), but affected little on the apoptosis of CRC cells (Additional file 2: Figure S2B).

\section{Nuclear translocation of JMJD2C lowered the histone} methylation level of MALAT1 promoter in CRC

Histone demethylase JMJD2C holds great potential of epigenetic regulating mechanism in tumor diseases [19-27], especially its important regulating effect on the promoter activity of targeted genes [28, 29]. By immunofluorescent staining assay, we found that, knockdown of JMJD2C could significantly decrease the nuclear accumulation of JMJD2C protein in CRC cells, while overexpression of JMJD2C could effectively elevate the distribution of JMJD2C protein in the nuclei of CRC cells (Fig. 3a, b).
Then, above results were further validated by the western blot detection (Fig. 3c, d).

Urged by above data, we next studied if nuclear JMJD2C could bind to the promoter of MALAT1 to affect the expression of MALAT1 by chromatin immunoprecipitation (ChIP) (Fig. 4a). Based on our ChIP assay, we found that JMJD2C could bind to the promoter of MALAT1, and regulate lower the histone methylation level of MALAT1 promoter in sites of $\mathrm{H} 3 \mathrm{~K} 9 \mathrm{~m} 3$ and $\mathrm{H} 3 \mathrm{~K} 36 \mathrm{~m} 3$, whether not only in HCT116 or but also in LoVo cells (Fig. 4b). Next, the luciferase reporter assay further confirmed that, JMJD2C could promote the promoter activity of MALAT1 gene (Fig. 4c, d). Moreover, the real-time PCR results also demonstrated that, JMJD2C could promote the transcriptional level of MALAT1 (Fig. 4e, f). All above results suggested that JMJD2C could translocate into the nuclei, regulate lower the histone methylation level of MALAT1 promoter, and elevate the expression of MALAT1.

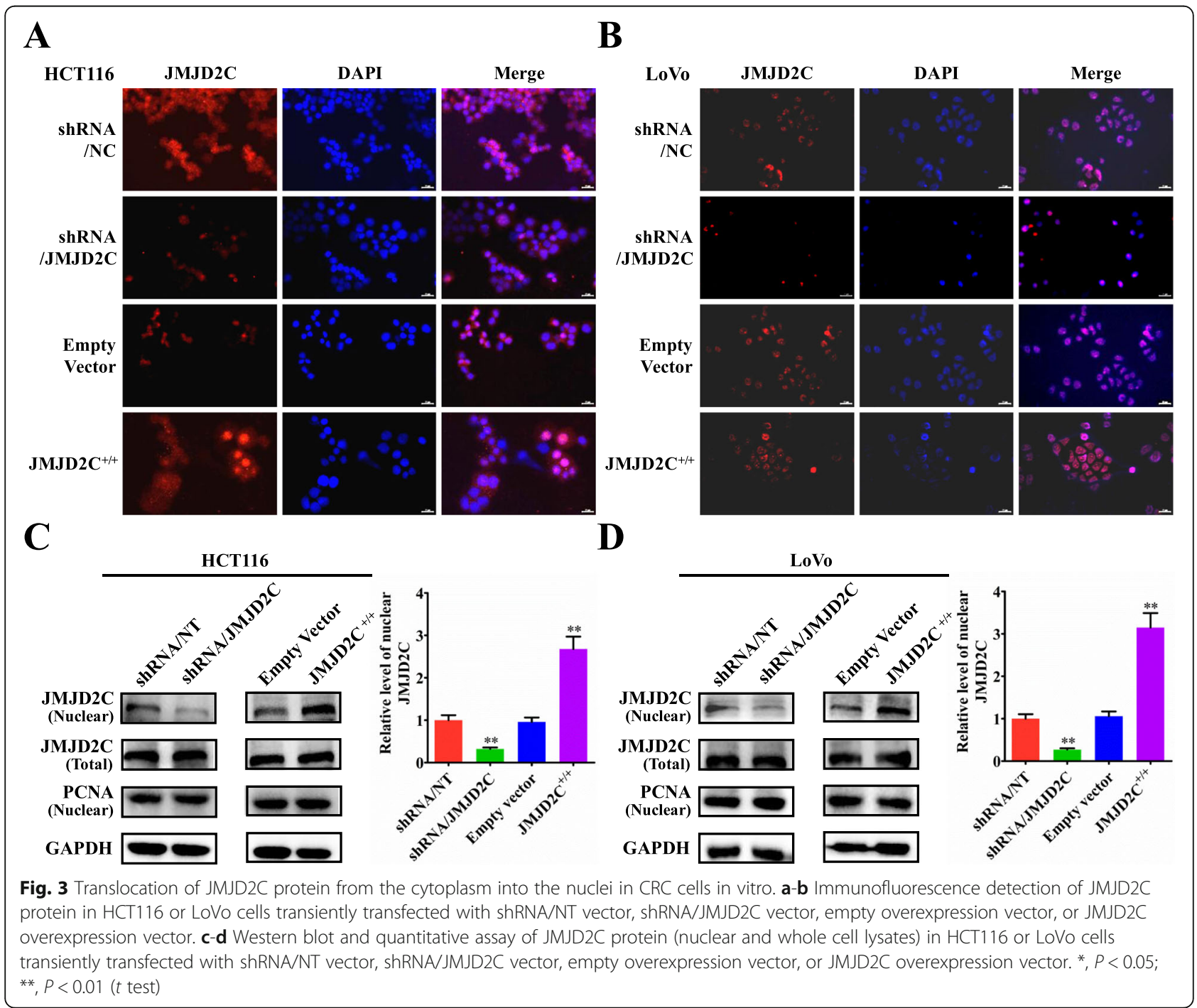




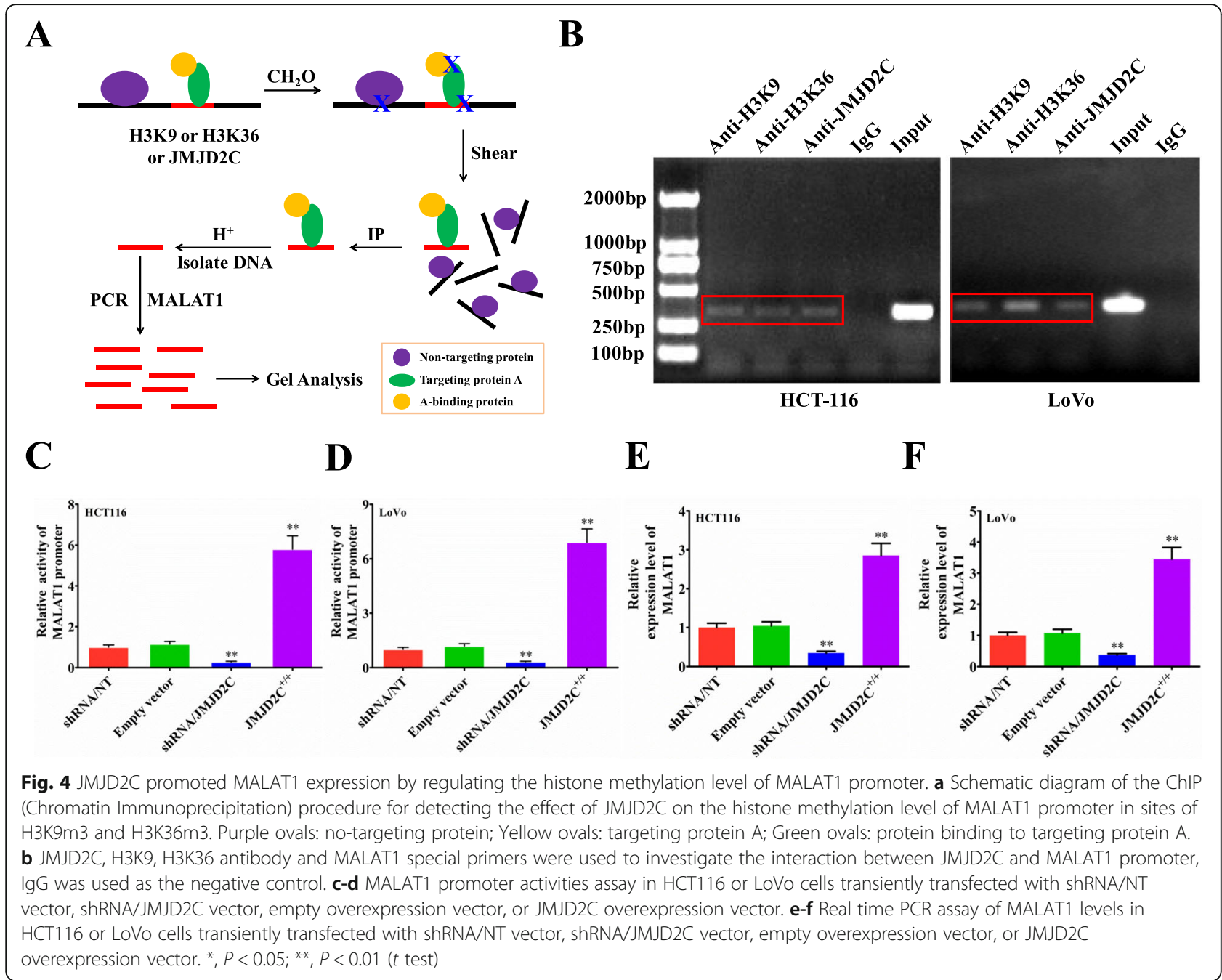

JMJD2C enhanced nuclear translocation of $\beta$-catenin and activated $\beta$-catenin signaling in CRC

Our previous studies have demonstrated that MALAT1 could enhance the $\beta$-catenin signaling pathway $[6,7]$; thus we further investigated the role of JMJD2C on regulating the expressions of $\beta$-catenin signaling pathway related proteins including $\beta$-catenin, c-Myc, and ITGBL1 (integrin $\beta$-like 1). By immunofluorescent staining assay, we found that, knockdown of JMJD2C could significantly decrease the nuclear accumulation of $\beta$ catenin protein in CRC cells, while overexpression of JMJD2C could effectively reverse the distribution of $\beta$ catenin in the nuclei of CRC cells (Fig. 5a, b). The following western blot results further confirmed the immunofluorescent staining results (Fig. 5c, d).

As we know, the nuclear $\beta$-catenin and other transcription factors such as LEF, TCF and BCL9 will function together to trans-activate downstream target genes [30]. Therefore, the luciferase reporter assay was further performed to observe the effect of JMJD2C on the activity of the LEF/TCF promoter. As expected, the final results confirmed the promoting effect of JMJD2C on the activity of the LEF/TCF promoter (Additional file 3: Figure S3). In addition, in Fig. 5c, d we also showed that, JMJD2C could enhance the expression of $\beta$-catenin signaling downstream targets including c-Myc and ITGBL1. All together, above data demonstrated JMJD2C enhanced the activity of $\beta$-catenin signaling pathway in CRC.

\section{JMJD2C promoted CRC lung metastasis in vivo}

In order to observe the in vivo effect of JMJD2C, HCT116shRNA/NT, HCT116-shRNA/JMJD2C, HCT116-empty vector, and HCT116- JMJD2C ${ }^{+/+}$cells were injected into nude mice (into the lateral tail vein), and the lung metastases images were observed by LB983 NIGHTOWL II (IVIS) system (Fig. 6a). As shown in Fig. 6b, knockdown of JMJD2C could significantly decrease the luciferase intensity of CRC cells in lung metastasis, while overexpression of JMJD2C could increase the luciferase intensity of CRC cells in lung metastasis. Additionally, the numbers of lung 


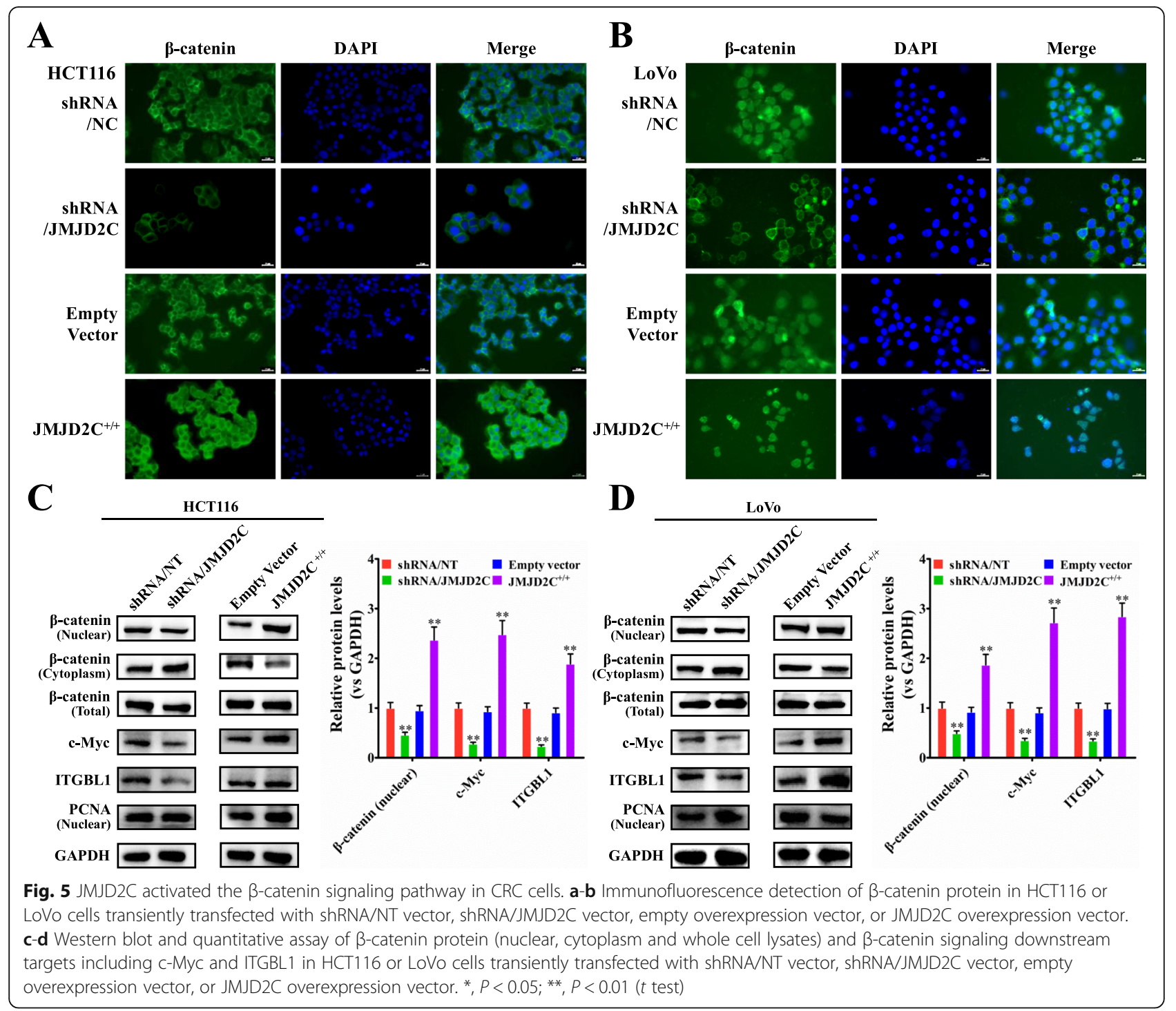

metastatic lesions were in accordance with the luciferase detecting results (Fig. 6c). Then, we detected the expression of JMJD2C protein in the lung metastatic lesions by immunohistochemical assay. The results showed the positive roles of JMJD2C in promoting CRC cells metastasis in vivo (Fig. 6d, e).

\section{JMJD2C elevated the expression of MALAT1 and}

$\beta$-catenin signaling related proteins in CRC lung metastasis mice models

Since the in vitro study has found the regulatory effect of JMJD2C on the expression of MALAT1 and $\beta$-catenin signaling related proteins, we next validated the results in vivo. Real-time PCR showed that, knockdown of JMJD2C could significantly decrease the expression of MALAT1 in the lung metastatic lesions, while overexpression of JMJD2C could significantly elevate the expression of MALAT1 in the lung metastatic lesions (Fig. 7a). Then, the western blot experiments further demonstrated that, knockdown of JMJD2C could significantly decrease the nuclear accumulation of $\beta$-catenin protein in the lung metastatic lesions, while overexpression of JMJD2C could effectively reverse the distribution of $\beta$-catenin in the nuclei of lung metastatic lesions (Fig. 7b, c). By immunohistochemical assay we further found that, knockdown of JMJD2C could significantly decrease the expression of $\beta$-catenin signaling downstream targets including c-Myc and ITGBL1 in the lung metastatic lesions, while overexpression of JMJD2C could significantly elevate the expression of c-Myc and ITGBL1 in the lung metastatic lesions (Fig. 7d, e).

\section{Discussion}

Histone demethylase JMJD2C has been reported to play crucial roles in the progression of colon cancer [19, 20], breast cancer [21-23], prostate cancer [24, 25], gastric 


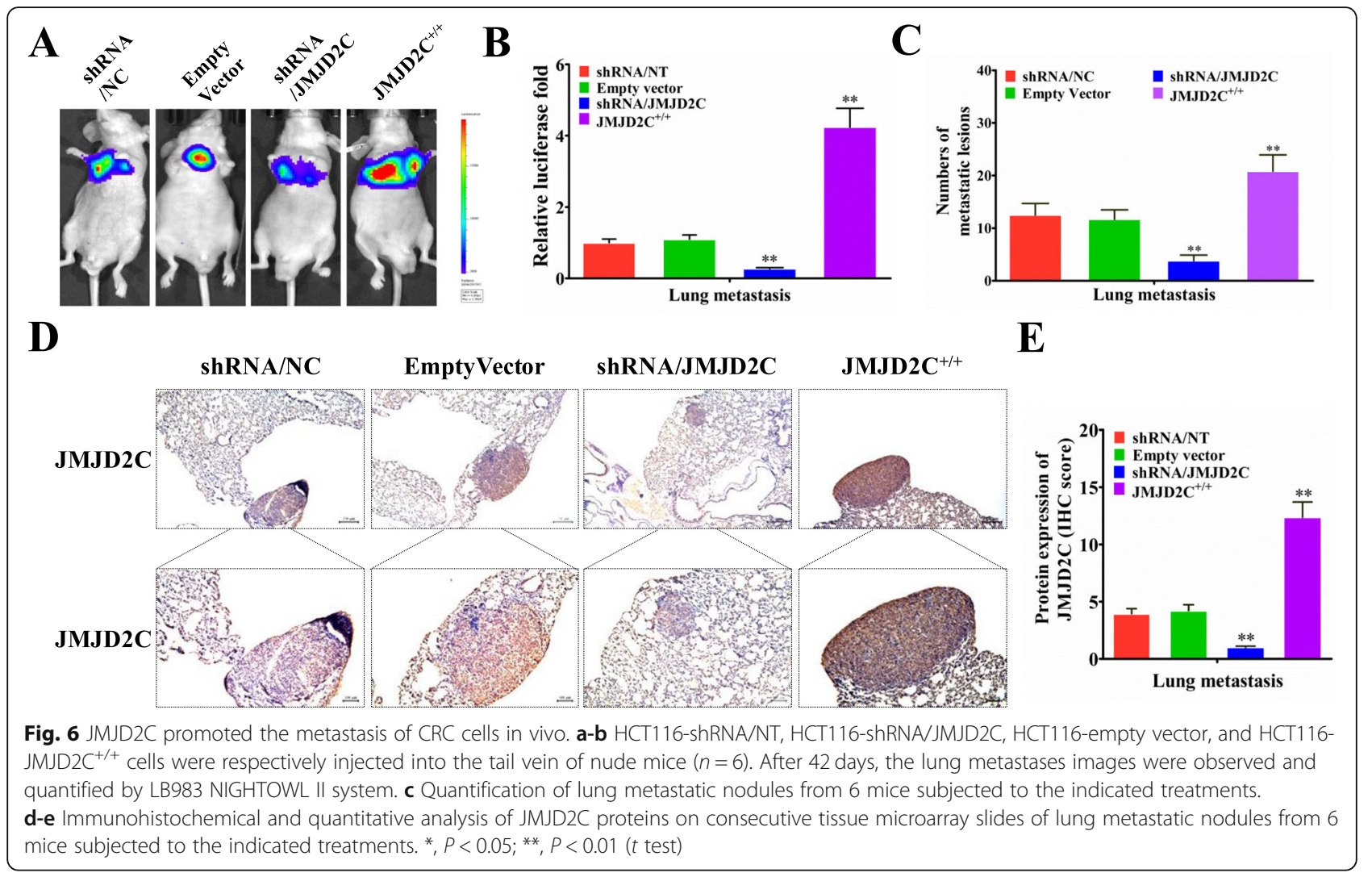

cancer [26], lung cancer [27] and so on, indicating that JMJD2C represents a promising anti-cancer target.

JMJD2C is mapped to human chromosome 9p24.1, and it has been proved to be a demethylase for H3K9 and H3K36 methylation [28, 29]. More evidences have shown that JMJD2C is a candidate oncogene both in normal biology and tumorigenic processes [31]. Ye et al [23] found that knockdown of JMJD2C inhibited the proliferation of breast cancer cells in vitro and in vivo. In addition, Kim et al firstly uncovered that JMJD2C was overexpressed in five colon cancer cell lines and especially associated closely with the growth of HCT116 cells, and JMJD2C might promote the survival of colon cancer cells and stimulate the proliferation of colon cancer cells via up-regulating the levels of Cyclin D1 and FRA1 [19]. These results revealed the important roles of JMJD2C in tumors through targeting different oncogenes or tumor suppressor genes.

Our previous studies have successfully demonstrated that MALAT1 could promote CRC metastasis through regulating $\beta$-catenin signaling pathway $[6,7]$. However, the upstream regulatory mechanism of MALAT1 is not well-elucidated. Based on bioinformatics analysis of the MALAT1 promoter, we found the potential binding sites for JMJD2C in the promoter of MALAT1, and hypothesized that, JMJD2C, the important histone demethylase, could influence the activity of MALAT1 promoter, thereby regulating MALAT1/ $\beta$-catenin signaling pathway and leading to the promotion of CRC metastasis.

In the presented paper, we demonstrated that the mRNA expression levels of JMJD2C in CRC metastatic lesions are significantly higher than the ones in primary lesions, and there is a positive correlation between JMJD2C mRNA expression and MALAT1 expression. Our previous studies have shown the promoting effect of MALAT1 on the proliferation and migration of CRC cells [6]. Currently, our results further suggested that JMJD2C can also promote the proliferation and migration of CRC cells in vitro. Nevertheless, the direct evidence about the interaction between JMJD2C and MALAT1 is not presented. Various studies have demonstrated the complicated biological function of JMJD2C. Ishimura et al has proved that overexpression of JMJD2C could up-regulate the expression of oncogene $\mathrm{Mdm} 2$ and lead to the decreased expression of tumor suppressor gene p53 [32]. Our data suggested that JMJD2C elevated the expression of MALAT1 and regulated the $\beta$-catenin signaling pathway, as well as the downstream gene expression including cMyc and ITGBL1. Further mechanism studies demonstrated that, JMJD2C could directly bind to the promoter region of MALAT1, lower the histone methylation level of MALAT1 promoter in the sites of H3K9me3 and H3K36me3, and enhance the promoter activity and the final expression of MALAT1. 

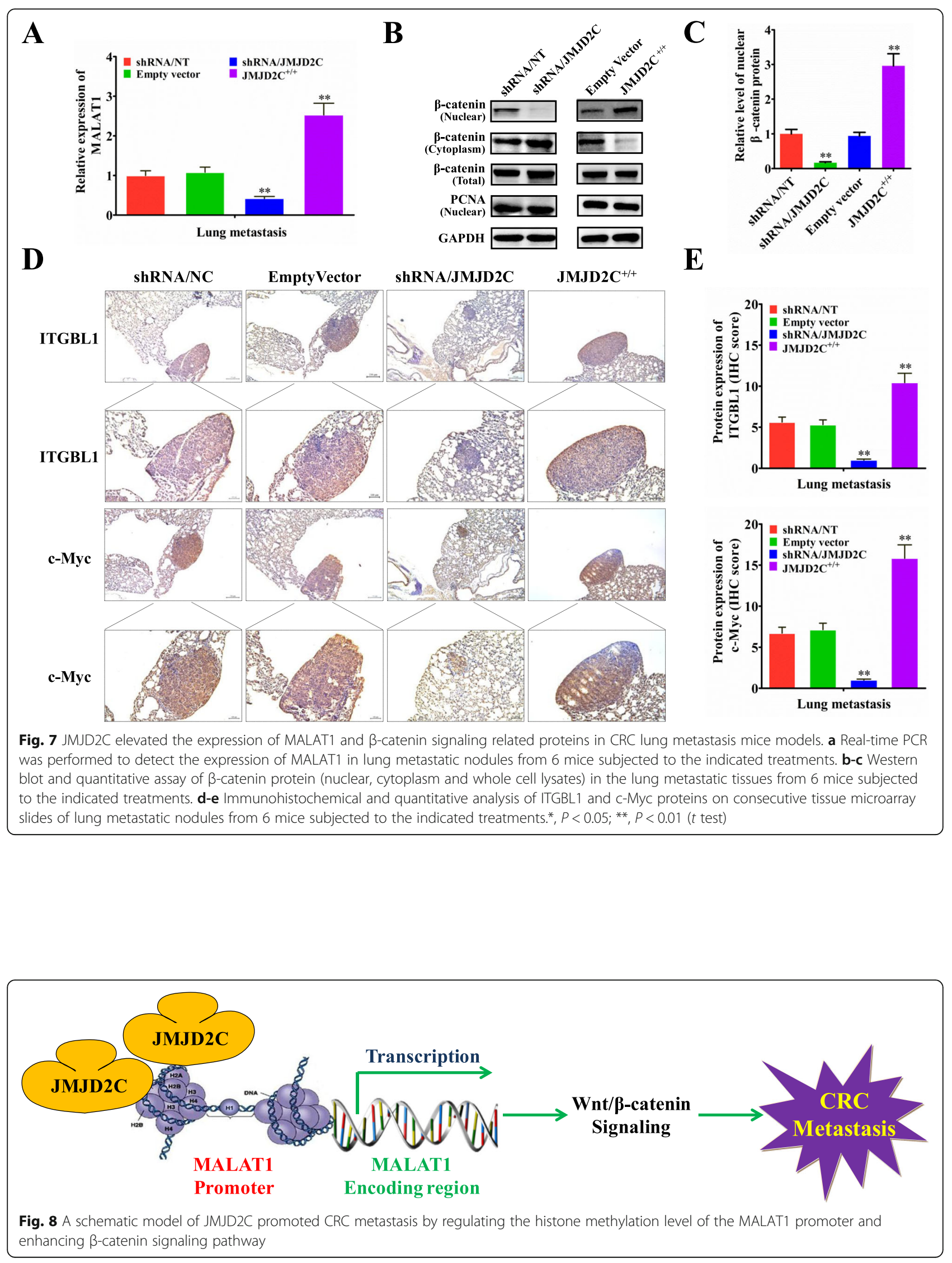
Ye et al has shown that knockdown of JMJD2C not only inhibited the breast tumor growth but also effectively blocked the lung metastasis in mice model [23]. In our study, by establishing the lung metastasis mice model, we found that JMJD2C could promote CRC metastasis in vivo. Moreover, in vivo, we also found that, JMJD2C could significantly elevate the expression of MALAT1 in the lung metastatic lesions, increase the nuclear accumulation of $\beta$-catenin protein, and elevate the protein expression of downstream targets including c-Myc and ITGBL1 in the lung metastatic lesions. These results further confirmed that JMJD2C could upregulate the MALAT1 expression and enhance the activity of $\beta$-catenin signaling pathway, which were in accordance with the results in vitro.

\section{Conclusion}

In summary, our findings imply that JMJD2C plays a vital role in the epigenetic regulation process. In details, JMJD2C could directly influence the expression of MALAT1 by regulating the histone methylation level of MALAT1 promoter in the sites of H3K9me3 and H3K36me3, enhancing the activity of $\beta$-catenin signaling pathway, and promoting CRC metastasis (Fig. 8). Thus, JMJD2C might be a useful target for the prevention and treatment of CRC metastasis, and the interaction between JMJD2C and MALAT1 could be considered as the points for drug development.

\section{Supplementary information}

Supplementary information accompanies this paper at https://doi.org/10 1186/s13046-019-1439-x.

Additional file 1: Figure S1. JMJD2C promoted the metastasis of CRC LoVo cells. a-c Real time PCR and western blotting were performed to confirm the gene silencing and overexpressing efficiency for JMJD2C. LoVo was transiently transfected with shRNA/NT vector, shRNA/JMJD2C vector, empty overexpression vector, or JMJD2C overexpression vector. d Migration assays of LoVo cells transfected with shRNA/NT, shRNA JMJD2C, empty vector, or JMJD2C overexpression vector, respectively. e Numbers of migrated cells are shown as mean $\pm S D ; n=3$. $^{*}, P<0.05$; **, $P<0.01$ ( $t$ test)

Additional file 2: Figure S2. JMJD2C promoted the proliferation of CRC HCT116 cells, but affected little on the apoptosis of the indicated cells. a MTT assay of HCT116 cells transfected with shRNA/NT, shRNA JMJD2C, empty vector, or JMJD2C overexpression vector, respectively. b Flow cytometry was performed to measure the apoptosis rates of HCT116 cells transfected with shRNA/NT, shRNA/JMJD2C, empty vector, or JMJD2C overexpression vector, respectively.

Additional file 3: Figure S3. JMJD2C enhanced the activity of the LEF/ TCF promoter. a-b LEF/TCF promoter activity assay in HCT116 and LoVo cells transfected with shRNA/NT, shRNA/JMJD2C, empty vector, or JMJD2C overexpression vector, respectively. ${ }^{*}, P<0.05 ;{ }^{* *}, P<0.01$ ( $t$ test).

\section{Abbreviations}

ChIP: Chromatin immunoprecipitation;; CRC: Colorectal cancer; DAB: Diaminobenzidine; HE: Hematoxylin and eosin* IHC: Immunohistochemistry; ITGBL1: Integrin $\beta$-like 1; JMJD2C: Jumonji domain-containing protein $2 \mathrm{C}$; IncRNAs: Long non-coding RNAs; MALAT1: Metastasis associated in lung adenocarcinoma transcript 1;
OS: Overall survival; PTBP-2: Polypyrimidine tract binding protein; SFPQ: PTBassociated splicing factor

\section{Acknowledgements}

We thank Professor Sizhi Paul Gao from Department of Medicine, Memorial Sloan Kettering Cancer Center.

\section{Authors' contributions}

QJ and QL conceived and designed the study and the experimental setup and wrote the manuscript. XW, CZ, QS, RL, ZH, RJ, YW, LZ, LY, and HS performed the experiments. QJ, QL and $\mathrm{HZ}$ analyzed the data. All authors read and approved the final version of the manuscript.

\section{Funding}

This work was supported by National Natural Science Foundation of China (No. 81830120 to QL), National Natural Science Foundation of China (No. 81573749 to QJ), National Natural Science Foundation of China (No. 81673783, No. 81874405 to XL), National Natural Science Foundation of China (No. 81603457 to ZFH), National Natural Science Foundation of China (No. 81874399 to HRZ), Science Foundation of Shanghai Committee of Science Project (No. 16401970500 to QL), Shanghai Rising-Star Program (No. 18QA1404100 to XL).

Availability of data and materials

All of the data and materials in this paper are available when requested.

Ethics approval and consent to participate

All the procedures involving human tumor biopsies were performed with the approval of the ethics committee of the Shuguang Hospital, Shanghai University of Traditional Chinese Medicine on samples from patients who had given written informed consent. All the animal protocols were approved by the Animal Care Commission of Shanghai University of Traditional

Chinese Medicine.

\section{Consent for publication}

Not applicable.

\section{Competing interests}

The authors declare that they have no competing interests.

\section{Author details}

${ }^{1}$ Department of Medical Oncology and Cancer Institute of Medicine, Shuguang Hospital, Shanghai University of Traditional Chinese Medicine, Shanghai, China. ${ }^{2}$ Department of Medical Oncology, Suzhou TCM Hospital Affiliated to Nanjing University of Chinese Medicine, Suzhou, China. ${ }^{3}$ Department of Medical Oncology, Longhua Hospital, Shanghai University of Traditional Chinese Medicine, Shanghai, China. ${ }^{4}$ Academy of Integrative Medicine, Shanghai University of Traditional Chinese Medicine, Shanghai, China.

Received: 12 August 2019 Accepted: 6 October 2019

Published online: 29 October 2019

References

1. Bray F, Ferlay J, Soerjomataram I, Siegel RL, Torre LA, Jemal A. Global cancer statistics 2018: GLOBOCAN estimates of incidence and mortality worldwide for 36 cancers in 185 countries. CA Cancer J Clin. 2018;68(6):394-424.

2. Watson AJ, Collins PD. Colon cancer: a civilization disorder. Dig Dis. 2011; 29(2):222-8

3. Siegel RL, Miller KD, Jemal A. Cancer statistics, 2017. CA Cancer J Clin. 2017; 67(1):7-30.

4. Bhan A, Soleimani M, Mandal SS. Long noncoding RNA and Cancer: a new paradigm. Cancer Res. 2017;77(15):3965-81.

5. Ji Q, Zhang L, Liu X, et al. Long non-coding RNA MALAT1 promotes tumour growth and metastasis in colorectal cancer through binding to SFPQ and releasing oncogene PTBP2 from SFPQ/PTBP2 complex. Br J Cancer. 2014; 111(4):736-48

6. Ji Q, Liu X, Fu X, et al. Resveratrol inhibits invasion and metastasis of colorectal cancer cells via MALAT1 mediated Wnt/beta-catenin signal pathway. PLoS One. 2013;8(11):e78700. 
7. Ji Q, Cai G, Liu X, et al. MALAT1 regulates the transcriptional and translational levels of proto-oncogene RUNX2 in colorectal cancer metastasis. Cell Death Dis. 2019;10(6):378.

8. Fan Y, Shen B, Tan M, et al. TGF-beta-induced upregulation of malat1 promotes bladder cancer metastasis by associating with suz12. Clin Cancer Res. 2014;20(6):1531-41.

9. Zhang LQ, Li QZ. Estimating the effects of transcription factors binding and histone modifications on gene expression levels in human cells. Oncotarget. 2017;8(25):40090-103.

10. Nordstrom L, Andersson E, Kuci V, et al. DNA methylation and histone modifications regulate SOX11 expression in lymphoid and solid cancer cells. BMC Cancer. 2015;15:273.

11. Su WX, Li QZ, Zuo YC, Zhang LQ. Association analysis between the distributions of histone modifications and gene expression in the human embryonic stem cell. Gene. 2016;575(1):90-100.

12. Helias C, Struski S, Gervais C, et al. Polycythemia vera transforming to acute myeloid leukemia and complex abnormalities including 9p homogeneously staining region with amplification of MLLT3, JMJD2C, JAK2, and SMARCA2. Cancer Genet Cytogenet. 2008;180(1):51-5.

13. Rui L, Emre NC, Kruhlak MJ, et al. Cooperative epigenetic modulation by cancer amplicon genes. Cancer Cell. 2010;18(6):590-605.

14. Northcott PA, Nakahara $Y$, Wu X, et al. Multiple recurrent genetic events converge on control of histone lysine methylation in medulloblastoma. Nat Genet. 2009;41(4):465-72.

15. Suikki HE, Kujala PM, Tammela TL, van Weerden WM, Vessella RL, Visakorpi $T$. Genetic alterations and changes in expression of histone demethylases in prostate cancer. Prostate. 2010;70(8):889-98.

16. Suzuki T, Miyata N. Lysine demethylases inhibitors. J Med Chem. 2011; 54(24):8236-50.

17. Hoffmann I, Roatsch M, Schmitt ML, et al. The role of histone demethylases in cancer therapy. Mol Oncol. 2012;6(6):683-703.

18. Kweider N, Fragoulis A, Rosen C, et al. Interplay between vascular endothelial growth factor (VEGF) and nuclear factor erythroid 2-related factor-2 (Nrf2): implications for preeclampsia. J Biol Chem. 2011;286:42863-72.

19. Kim TD, Fuchs JR, Schwartz E, et al. Pro-growth role of the JMJD2C histone demethylase in HCT-116 colon cancer cells and identification of curcuminoids as JMJD2 inhibitors. Am J Transl Res. 2014;6(3):236-47.

20. Kim TD, Shin S, Berry WL, Oh S, Janknecht R. The JMJD2A demethylase regulates apoptosis and proliferation in colon cancer cells. J Cell Biochem. 2012;113(4):1368-76.

21. Luo W, Chang R, Zhong J, Pandey A, Semenza GL. Histone demethylase JMJD2C is a coactivator for hypoxia-inducible factor 1 that is required for breast cancer progression. Proc Natl Acad Sci U S A. 2012;109(49):E3367-76.

22. Liu G, Bollig-Fischer A, Kreike B, et al. Genomic amplification and oncogenic properties of the GASC1 histone demethylase gene in breast cancer. Oncogene. 2009;28(50):4491-500.

23. Ye Q, Holowatyj A, Wu J, et al. Genetic alterations of KDM4 subfamily and therapeutic effect of novel demethylase inhibitor in breast cancer. Am J Cancer Res. 2015;5(4):1519-30.

24. Kahl P, Gullotti L, Heukamp LC, et al. Androgen receptor coactivators lysinespecific histone demethylase 1 and four and a half LIM domain protein 2 predict risk of prostate cancer recurrence. Cancer Res. 2006;66(23):11341-7.

25. Wissmann M, Yin N, Muller JM, et al. Cooperative demethylation by JMJD2C and LSD1 promotes androgen receptor-dependent gene expression. Nat Cell Biol. 2007;9(3):347-53.

26. Xu Y, Yao HB, Jin Y, Ma YY, Wang HJ, Shao QS. Expressions of Jumonji domain containing protein $2 \mathrm{C}$ and hypoxia-inducible factor-1alpha in gastric carcinoma and their clinical significance. Zhonghua Yi Xue Za Zhi. 2013;93(42):3375-8.

27. Li N, Jiang D. Jumonji domain containing $2 \mathrm{C}$ promotes cell migration and invasion through modulating CUL4A expression in lung cancer. Biomed Pharmacother. 2017:89:305-15.

28. Hillringhaus $L$, Yue WW, Rose NR, et al. Structural and evolutionary basis for the dual substrate selectivity of human KDM4 histone demethylase family. J Biol Chem. 2011;286(48):41616-25.

29. Wang Z, Zang C, Rosenfeld JA, et al. Combinatorial patterns of histone acetylations and methylations in the human genome. Nat Genet. 2008;40(7): 897-903.

30. Zhao JJ, Lin JH, Zhu D, et al. miR-30-5p functions as a tumor suppressor and novel therapeutic tool by targeting the oncogenic Wnt/B-catenin/BCL9 pathway. Cancer Res. 2014;74(6):1801-13.
31. Burton A, Azevedo C, Andreassi C, Riccio A, Saiardi A. Inositol pyrophosphates regulate JMJD2C-dependent histone demethylation. Proc Natl Acad Sci U S A. 2013;110(47):18970-5.

32. Ishimura A, Terashima M, Kimura $\mathrm{H}$, et al. Jmjd2c histone demethylase enhances the expression of Mdm2 oncogene. Biochem Biophys Res Commun. 2009;389(2):366-71.

\section{Publisher's Note}

Springer Nature remains neutral with regard to jurisdictional claims in published maps and institutional affiliations.
Ready to submit your research? Choose BMC and benefit from:

- fast, convenient online submission

- thorough peer review by experienced researchers in your field

- rapid publication on acceptance

- support for research data, including large and complex data types

- gold Open Access which fosters wider collaboration and increased citations

- maximum visibility for your research: over $100 \mathrm{M}$ website views per year

At BMC, research is always in progress.

Learn more biomedcentral.com/submissions 